\title{
Azole-resistant invasive aspergillosis in a patient with acute myeloid leukaemia in Germany
}

A Hamprecht (axel.hamprecht@uk-koeln.de) ${ }^{1}$, D Buchheidt ${ }^{2}$, J J Vehreschild 3 , 0 A Cornely3,4, B Spiess ${ }^{2}$, G Plum ${ }^{1}$, T V Halbsguth 3 ,

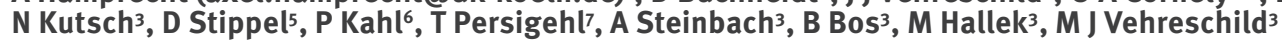

1. Institute for Medical Microbiology, Immunology and Hygiene, University of Cologne, Cologne, Germany

2. Third Department of Internal Medicine, Haematology and Oncology, Mannheim University Hospital, University of Heidelberg, Mannheim, Germany

3. First Department of Internal Medicine, University of Cologne, Cologne, Germany

4. Clinical Trials Centre Cologne, ZKS Köln (BMBF 01KN1106), University of Cologne, Cologne, Germany

5. Department of General, Visceral and Cancer Surgery, University of Cologne, Germany

6. Institute of Pathology, University Hospital Cologne, Cologne, Germany

7. Department of Radiology, University Hospital Cologne, Cologne Germany

Citation style for this article:

Hamprecht A, Buchheidt D, Vehreschild JJ, Cornely OA, Spiess B, Plum G, Halbsguth TV, Kutsch N, Stippel D, Kahl P, Persigehl T, Steinbach A, Bos B, Hallek M, Vehreschild MJ. Azole-resistant invasive aspergillosis in a patient with acute myeloid leukaemia in Germany. Euro Surveill. 2012;17(36):pii=20262. Available online: http://www.eurosurveillance.org/ViewArticle.aspx?Articleld=20262

We report the first culture-proven case of invasive aspergillosis (IA) caused by azole-resistant Aspergillus fumigatus in a patient with acute myeloid leukaemia in Germany. IA presented as breakthrough infection under posaconazole prophylaxis. Analysis of the resistance mechanism revealed the TR/L98 $\mathrm{H}$ mutation in the cyp51A gene, which indicates an environmental origin of the strain. This case underscores the need for monitoring azole resistance in Aspergillus spp. and for routine susceptibility testing of moulds.

\section{Background}

Invasive aspergillosis (IA) is the most frequent invasive fungal disease (IFD) in patients with haematological malignancies and in those undergoing allogeneic stem cell transplantation, with $A$. fumigatus being its prime causative agent. Azole antifungals are recommended as first-line treatment and prophylaxis of IA in high risk patients in most international guidelines. Azole resistance is observed in patients with long-term azole therapy, but also in azole-naïve patients, suggesting an environmental origin for the latter group. Resistance to azole antifungals in clinical $A$. fumigatus isolates was reported from the Netherlands, the United Kingdom, France, the United States and recently China and India and has been associated with a mortality of up to $88 \%$ [1-4]. In Europe, resistant clinical strains were mostly isolated from patients with chronic pulmonary disease and prior long-term antifungal therapy, e.g. patients with allergic bronchopulmonary aspergillosis, chronic pulmonary aspergillosis or cystic fibrosis $[5,6]$. In patients with haematological malignancies, azole resistance has rarely been reported [7].

\section{Case report}

A 35 year-old male patient was admitted to the University Hospital of Cologne with a first diagnosis of acute myeloid leukaemia (AML) in March 2012. He was started on remission-induction chemotherapy on the same day (day o) and he received oral posaconazole prophylaxis (200 $\mathrm{mg}$ three times a day) from day 5 . The patient was included into the ongoing study on the epidemiology of invasive aspergillosis and resistance patterns of Aspergillus spp. (SEPIA study) for surveillance of patients with acute leukaemia. A baseline chest computed tomography scan (CT) showed no signs of IFD. A consecutive episode of febrile neutropenia (day 13) was treated with empirical broad-spectrum antibiotics, but fever persisted.

A PCR from a throat swab revealed respiratory syncytial virus (RSV) as the possible origin of fever, cough and dyspnoea. A chest CT scan showing bilateral patchy ground glass opacities was compatible with this diagnosis. However, a broncho-alveolar lavage (BAL) performed on day 25 after admission revealed an elevated galactomannan (GM) index of $\mathbf{2 2 . 5}$ from BAL fluid (norm: $<0.9$ ), which is indicative of IA. Serum levels of GM increased from 0.4 to 1.3 (norm: $<0.5$ ), even though posaconazole levels were adequate $(2.01 \mathrm{mg} / \mathrm{L}$; standard range not defined).

A novel PCR assay [8] performed from the same BAL fluid was positive for $A$. fumigatus and the TR/L98H mutation of cyp51A. In addition, A. fumigatus sensu stricto was isolated in culture, with elevated minimum inhibitory concentrations (MICs) for voriconazole (2 $\mathrm{mg} / \mathrm{L}$ ) and posaconazole (0.5 mg/L, Table 1). Treatment was switched to intravenous liposomal amphotericin B (LAmB) on day 35 at a dose of $3 \mathrm{mg} / \mathrm{kg}$.

A follow-up chest CT scan on day 43, seven days after recovery from neutropenia, showed multiple larger lung nodules with air crescent sign in the right upper and left lower lobe and was strongly suggestive of pulmonary IA responding to $L A m B$. In addition, a new 
TABLE

Minimum inhibitory concentrations of Aspergillus fumigatus isolate from a patient with acute myeloid leukaemia, and EUCAST clinical breakpoints [9], Germany 2012

\begin{tabular}{|l|c|c|c|}
\hline Antifungal drug & $\begin{array}{c}\text { Testing } \\
\text { method }\end{array}$ & MIC $[\mathrm{mg} / \mathrm{L}]$ & $\begin{array}{c}\text { EUCAST } \\
\text { breakpoint } \\
\text { S/R }\end{array}$ \\
\hline Voriconazole & EUCAST & 2 & - \\
\hline Posaconazole & EUCAST & 0.5 & $\leq 0.12 />0,25$ \\
\hline Itraconazole & EUCAST & $>16$ & $\leq 1 />2$ \\
\hline Amphotericin B & EUCAST & 0.5 & $\leq 1 />2$ \\
\hline Caspofungin & Etest & 0.032 & - \\
\hline
\end{tabular}

EUCAST: The European Committee on Antimicrobial Susceptibility Testing; MIC: minimum inhibitory concentration; R: resistant; S: susceptible. splenic abscess was described. Splenectomy was performed on day 61. Culture of spleen tissue remained negative for bacteria and fungi, but the histological workup revealed abundant hyphae compatible with IA.

On day 140 , the patient had recovered and was in complete remission from AML.

\section{Phenotypic identification, molecular identification and resistance testing}

A. fumigatus sensu stricto was identified by morphological and molecular characteristics. DNA of the $A$. fumigatus strain was isolated using the QIAamp DNA mini kit (Qiagen, Hilden, Germany). Molecular identification was done by sequencing the internal transcribed spacer (ITS) and partial beta-tubulin gene of the isolate [10]. Susceptibility testing for azole antifungals and amphotericin $B$ was performed by microdilution testing according to EUCAST [11]. Caspofungin MIC was determined by Etest (Biomérieux, Nürtingen, Germany) according to the manufacturer's recommendation. Presence of the $\mathrm{L} 98 \mathrm{H}$ mutation and the $34 \mathrm{bp}$ tandem repeat (TR) was evidenced by sequencing the complete cyp51A gene and its promotor region [12, 13]. Direct PCR from BAL fluid for the detection of $A$. fumigatus and azole-resistance genes was carried out as previously described [8].

\section{Discussion}

A. fumigatus resistant to itraconazole and posaconazole bearing the TR/L98H mutation was identified in a patient with AML from Germany after only 14 days of azole treatment. This short duration of exposure as well as the TR/L98H mutation suggests an infection with a strain of environmental origin. The patient had not visited the neighbouring Netherlands within the last 12 months, where TR/L98H has frequently been described in azole-resistant $A$. fumigatus strains. Therefore, his respiratory tract may have been colonised with a TR/ $\mathrm{L} 98 \mathrm{H}$ mutant strain from Germany prior to development of IA.

Azole resistance is usually mediated by different point mutations in the cyp51A gene, coding for 14 alphademethylase, a component of the ergosterol synthesis pathway and target of azole antifungals. The frequency of different resistance mechanisms varies between study locations, patient populations and countries. In the United Kingdom, several different mutations are commonly found, such as M220 or G54 [5]. In the Netherlands, the TR/L98H mutation prevails, which was detected in more than $90 \%$ of itraconazole resistant strains [4]. TR/L98H mutant strains are reported to be of environmental origin and as a consequence of the use of 14 alpha-demethylase inhibitors as fungicides in agriculture [14]. In these strains, a leucine to histidine substitution $(\mathrm{L} 98 \mathrm{H})$ is associated with a 34 -bp TR in the promotor region [4]. According to recently published data, this resistance mechanism is spreading across Europe [15].

Considering that prophylaxis and first-line treatment of IA is usually based on azoles, further spread of resistant isolates could jeopardise the effectiveness of prophylactic strategies and targeted treatment.

Susceptibility testing of fungi, especially of moulds, is not carried out on a routine basis in most microbiology laboratories. In comparison to susceptibility testing of bacteria, testing of moulds is more laborious, it cannot be automated today and requires sound mycological knowledge. Breakpoints for antifungals in Aspergillus spp. were published only recently and for a limited number of species and drugs [9]. Detection of azole resistance can be challenging, as some isolates have only slightly elevated or normal MICs for posaconazole and voriconazole. Therefore, itraconazole should be routinely tested on all isolates from patients needing antifungal therapy, because cyp $51 \mathrm{~A}$ mutant strains usually show the highest MIC to this compound.

Furthermore, reliable detection of $A$. fumigatus and cyp $51 \mathrm{~A}$ mutations can be achieved directly from clinical samples by PCR assays with subsequent sequencing $[8,16]$, offering faster and culture independent recognition of resistance.

More data on the susceptibility of Aspergillus spp., both from environmental and clinical samples is needed to reliably describe the epidemiology of azole resistance in Europe. Susceptibility testing of Aspergillus spp. should be routinely carried out in clinical laboratories to detect resistant strains and prevent therapeutic failure.. 


\section{Acknowledgments}

The patient was registered in the SEPIA Study (A Study on the Epidemiology of Invasive Aspergillosis and Resistance Patterns of Aspergillus spp.), funded by a grant from the Deutsche José Carreras Leukämie-Stiftung e.V.

Conflicts of Interest

A. Hamprecht has received a travel grant from Astellas. D. Buchheidt has received research grants from Gilead Sciences and Pfizer and served on the speakers' bureaux of Astellas, Gilead Sciences, Merck Sharp Dohme/ Merck and Pfizer. J.J. Vehreschild has received research grants from Astellas, Essex/Schering-Plough, Infectopharm, and Pfizer; and served on the speakers' bureau of Astellas, Essex/ScheringPlough, and Merck Sharp Dohme/ Merck. O.A. Cornely has received research grants from Astellas, Basilea, Bayer, Genzyme, Gilead, Merck/Schering, Merck/Serono, Optimer, and Pfizer, has been a consultant to Astellas, Basilea, F2 G, Gilead, Merck/Schering, Optimer, and Pfizer. A. Steinbach has received a research grant from MSD and served on the speakers' bureau of Gilead Sciences. M.J.G.T. Vehreschild has served on the speakers' bureau of Schering-Plough/ Essex, Pfizer, MSD and Gilead Sciences. She has received a research grant from $3 \mathrm{M}$.

\section{References}

1. Chowdhary A, Kathuria S, Randhawa HS, Gaur SN, Klaassen $\mathrm{CH}$, Meis JF. Isolation of multiple-triazole-resistant Aspergillus fumigatus strains carrying the TR/L98H mutations in the cyp51A gene in India. J Antimicrob Chemother. 2012;67(2):362-6.

2. Lockhart SR, Frade JP, Etienne KA, Pfaller MA, Diekema DJ, Balajee SA. Azole resistance in Aspergillus fumigatus isolates from the ARTEMIS global surveillance study is primarily due to the TR/L98H mutation in the cyp51A gene. Antimicrob Agents Chemother. 2011;55(9):4465-8

3. Mayr A, Lass-Flörl C. Epidemiology and antifungal resistance in invasive Aspergillosis according to primary disease: review of the literature. Eur J Med Res. 2011;16(4):153-7.

4. van der Linden JW, Snelders E, Kampinga GA, Rijnders BJ, Mattsson E, Debets-Ossenkopp YJ, et al. Clinical implications of azole resistance in Aspergillus fumigatus, The Netherlands, 2007-2009. Emerg Infect Dis. 2011;17(10):1846-54.

5. Howard SJ, Cerar D, Anderson MJ, Albarrag A, Fisher MC, Pasqualotto AC, et al. Frequency and evolution of Azole resistance in Aspergillus fumigatus associated with treatment failure. Emerg Infect Dis. 2009;15(7):1068-76.

6. Mortensen KL, Jensen RH, Johansen HK, Skov M, Pressler T, Howard SJ, et al. Aspergillus species and other molds in respiratory samples from patients with cystic fibrosis: a laboratory-based study with focus on Aspergillus fumigatus azole resistance. J Clin Microbiol. 2011;49(6):2243-51.

7. Alanio A, Sitterle E, Liance M, Farrugia C, Foulet F, Botterel $\mathrm{F}$, et al. Low prevalence of resistance to azoles in Aspergillus fumigatus in a French cohort of patients treated for haematological malignancies. J Antimicrob Chemother. 2011;66(2):371-4

8. Spiess B, Seifarth W, Merker N, Howard SJ, Reinwald M, Dietz A, et al. Development of Novel PCR Assays To Detect Azole Resistance-Mediating Mutations of the Aspergillus fumigatus cyp51A Gene in Primary Clinical Samples from Neutropenic Patients. Antimicrob Agents Chemother. 2012 ;56(7):3905-10.

9. European Committee on Antimicrobial Susceptibility Testing (EUCAST) Antifungal Agents - breakpoint tables for interpretation of MICS. 2012; version 4.1. Available from: http://www.eucast.org/fileadmin/src/media/PDFs/ EUCAST_files/AFST/Antifungal_breakpoints_v_4.1.pdf

10. Glass NL, Donaldson GC. Development of primer sets designed for use with the PCR to amplify conserved genes from filamentous ascomycetes. Appl Environ Microbiol. 1995;61(4):1323-30.

11. Rodriguez-Tudela IL, Arendrup MC, Arikan S, Barchiesi F, Bille J, Chryssanthou E, et al. EUCAST DEFINITIVE DOCUMENT E.DEF 9.1: Method for the determination of broth dilution minimum inhibitory concentrations of antifungal agents for conidia forming moulds. EUCAST EDEF 91. 2008:1-13.

12. Chen J, Li H, Li R, Bu D, Wan Z. Mutations in the cyp51A gene and susceptibility to itraconazole in Aspergillus fumigatus serially isolated from a patient with lung aspergilloma. J Antimicrob Chemother. 2005;55(1):31-7.

13. Mellado E, Diaz-Guerra TM, Cuenca-Estrella M, RodriguezTudela IL. Identification of two different 14-alpha sterol demethylase-related genes (cyp51A and cyp 51B) in Aspergillus fumigatus and other Aspergillus species. J Clin Microbiol. 2001;39(7):2431-8.

14. Snelders E, Camps SM, Karawajczyk A, Schaftenaar G, Kema $\mathrm{GH}$, van der Lee HA, et al. Triazole fungicides can induce crossresistance to medical triazoles in Aspergillus fumigatus. PLoS One. 2012;7(3):e31801.

15. Camps SM, Rijs AJ, Klaassen CH, Meis JF, O'Gorman CM, Dyer PS, et al. Molecular Epidemiology of Aspergillus fumigatus Isolates Harboring the TR34/L98H Azole Resistance Mechanism. J Clin Microbiol. 2012;50(8):2674-80.

16. Denning DW, Park S, Lass- Flörl C, Fraczek MG, Kirwan M, Gore R, et al. High-frequency triazole resistance found In nonculturable Aspergillus fumigatus from lungs of patients with chronic fungal disease. Clin Infect Dis. 2011;52(9):1123-9. 